Jasmina Mahmutović ${ }^{1}$

Universität Sarajevo

Philosophische Fakultät

(Doktorandin)
удК 811.112.2'37

DOI https://doi.org/10.18485/zivjez.2017.37.1.3

Оригинални научни рад

\title{
NATUR UND KULTUR ALS BILDSPENDER FÜR DIE KONZEPTUALISIERUNG DER WIRTSCHAFT
}

Mit diesem Beitrag soll gezeigt werden, wie die komplexe Domäne Wirtschaft durch den Gebrauch von Metaphern aus den Bereichen Natur und Kultur kognitiv bewältigt wird. Im Fokus der Analyse steht die komplexitätsreduzierende, veranschaulichende und handlungsanleitende Rolle der Metapher. Es interessieren die mit den jeweiligen metaphorischen Szenarien mitgelieferten Deutungen der Wirklichkeit und nahegelegten Handlungen. Ziel ist es, herauszufinden, ob wirtschaftliche Abläufe als naturgegeben oder als vom Menschen geschaffen und somit beinflussbar betrachtet werden. Gleichermaßen interessieren die sprachlich erzeugten Menschenbilder. Den theoretischen und methodischen Rahmen liefert die Konzeptuelle Metapherntheorie, die davon ausgeht, dass sich mentale Strukturen über die Analyse des Sprachgebrauchs systematisch erfassen lassen.

Schlüsselwörter: Natur, Kultur, Wirtschaft, Metaphern, Menschenbilder.

\section{Gegenstand und Methode}

Im Fokus der vorliegenden Arbeit stehen die jeweils durch den Gebrauch von Metaphern aus den Bereichen Natur und Kultur erzeugten Vorstellungen über die Funktionsweise der Wirtschaft. Den Gegenstand des Interesses bildet nicht nur die Deutung hochkomplexer und schwerverständlicher ökonomischer Abläufe, sondern auch die sprachlich entworfenen Menschenbilder und die mit dem jeweiligen metaphorischen Szenario nahegelegte Handlung. Es wird davon ausgegangen, dass sich das Wissen über abstrakte

1 jasmina12mahmutovic@hotmail.com 
Jasmina Mahmutović

und hochkomplexe wirtschaftliche Phänomene über die Analyse des Sprachgebrauchs rekonstruieren lässt.

Wirtschaft bestimmt den menschlichen Alltag wie kaum eine andere Domäne und ist für jeden Einzelnen unumgehbar. Überall ist der Mensch als „Wirtschaftsbürger" (Hebel 1987: 61), sei es als Verbraucher, Arbeitnehmer, Steuerzahler oder Bankkunde in das Wirtschaftsgeschehen eingebunden. Wirtschaft ist jedoch ein Bereich, bei dem die Gegenstände, über die kommuniziert wird, nicht in materieller Weise zuhanden sind. Insofern ist die Rolle der Sprache in dieser Domäne von zentraler Bedeutung. Der Großteil unseres Wissens über die Wirtschaft wird mit Sprache erst konstituiert. Vor allem im Dienstleistungsbereich, der den Löwenanteil der heutigen Gesamtwirtschaft ausmacht, wird nicht mit konkreten, haptischen Produkten gehandelt, sondern mit abstrakten, sprachlich geschaffenen Gütern. Insofern lässt sich mit Coulmas (1992: 123) festhalten, dass Sprache und Geld die einzigen Mittel sind, mit denen in der Wirtschaft gehandelt wird. Umgekehrt bedeutet das für eine linguistische Analyse: Wenn Wirtschaft ein sprachlich erzeugtes Konstrukt ist, dann muss sich dieses Phänomen anhand der Analyse des Sprachgebrauchs rekonstruieren lassen.

\section{Das Textkorpus}

Das Textkorpus für die vorliegende Untersuchung besteht aus 156 Texten, die aus drei überregionalen bundesdeutschen Leitmedien stammen und ein relativ breites politisches Spektrum abdecken: dem Nachrichtenmagazin Der Spiegel sowie den Wochenzeitungen Die Zeit und Frankfurter Allgemeine Sonntagszeitung. Der Erscheinungszeitraum der analysierten Texte liegt zwischen dem 16. September 2008 und dem 20. September 2009. Es handelt sich um einen Zeitraum, in dem Deutschland von der Wirtschaftskrise 2008/09 erfasst war. Der gewählte Untersuchungszeitraum erscheint insofern besonders gut für die Analyse der Wirtschaftsmetaphorik geeignet, als das Thema Wirtschaft in dem abgedeckten Zeitraum im Mittelpunkt der öffentlich-politischen Diskussion stand und von den Medien breit thematisiert wurde. 
NATUR UND KULTUR ALS BILDSPENDER FÜR DIE KONZEPTUALISIERUNG...

\section{Theorie und Methode}

Theoretisch und methodisch bewegt sich diese Arbeit im Rahmen der Konzeptuellen Metapherntheorie ${ }^{2}$. Diese Theorie sieht in der Metapher kein rein sprachliches, sondern ein primär kognitives Phänomen, das in allen Lebensbereichen unser Denken, Reden und Handeln strukturiert (Lakoff et al. 2011: 12).

Das Wesen der Metapher besteht darin, daß wir durch sie eine Sache oder einen Vorgang in Begriffen einer anderen Sache bzw. eines anderen Vorgangs verstehen und erfahren können. (ebd.)

Metaphern finden sich primär dort, wo es um Grenzgebiete menschlicher Erfahrung geht. Das gilt besonders für abstrakte Sachverhalte und höchstkomplexe wissenschaftliche Vorgänge, für die kein sinnlich fassbares Referenzobjekt vorhanden ist (Schwarz-Friesel 2015: 144). Bei dem hier untersuchten abstrakten Bereich Wirtschaft ist die Metapher nicht als stilistischer Schmuck, sondern als eine "kognitive Notwendigkeit" (Jäkel 2003), (Ortony 1975) zu verstehen. Die komplexe Wirtschaftswelt, die nicht „mit bloßen Augen und Ohren“ (Luhmann 1999: 9) erfasst werden kann, wird mithilfe von Metaphern in konkrete, bildhafte Vorstellungen übersetzt und somit verständlich gemacht. Die These von der komplexitätsreduzierenden und veranschaulichenden Rolle der Metapher bildet den Ausgangspunkt für die nachfolgende Untersuchung.

Die Konzeptuelle Metapherntheorie geht davon aus, dass kognitive Metaphern über die Analyse ihrer sprachlichen Realisierungen systematisch erfasst und rekonstruiert werden können (Lakoff 1993: 244). Der lexikalische Ansatz wird auch in dieser Arbeit angewandt. Bei dem Analyseverfahren wird von den konkreten sprachlichen Ausdrücken ausgegangen und schrittweise hin zu den abstrakten konzeptuellen Metaphern geschlossen. Die im Textmaterial eruierten Natur- und Kulturmetaphern werden nach semantischen Ähnlichkeiten klassifiziert und in Metaphernbereiche ${ }^{3}$ zusammengefasst. Abschließend werden die Metaphernbereiche anhand dominanter Belege interpretiert.

2 Die "Conceptual Theory of Metaphor" wurde von Lakoff und Johnson begründet.

3 Ein Metaphernbereich ist eine „systematische Kopplung eines Herkunfts- an einen Zielbereich“ (Böke 1996: 444). 


\subsection{Die Bildspender Natur und Kultur}

Wo von abstrakten Phänomenen die Rede ist, sind Metaphern nicht weit. Der menschliche Körper und sein unmittelbares Umfeld gelten in den kognitiven Metapherntheorien als die zwei wichtigsten Bereiche, die zur Erzeugung von Metaphern herangezogen werden. Das Interesse dieser Arbeit richtet sich auf das menschliche Umfeld als einem zentralen Bildspender bei der metaphorischen Darstellung der Wirtschaft. Das menschliche Umfeld wird zum Zweck der Beschreibung der relevanten Metaphern in das Gegensatzpaar Natur und Kultur eingeteilt. „Will man die Welt beschreiben“, schreibt Wolfgang Marschall (1993), „,so reichen dafür die Begriffe Natur und Kultur" (Hansen 2003: 19). Im Folgenden wird es zunächst darum gehen, das hier zugrundeliegende Begriffspaar Natur und Kultur zu präzisieren. Im Anschluss daran werden die diskursrelevanten Metaphern nach dem Oppositionspaar Natur und Kultur klassifiziert und anhand prägnanter Beispiele besprochen.

Ob Natur oder Kultur am Werke ist, zieht praktische Konsequenzen für die Auffassung der Funktionsweise der Wirtschaft nach sich und legt unterschiedliche Handlungsweisen nahe. Der Bereich der Natur ist nicht vom Menschen gemacht und bleibt seinem Einfluss entzogen, derjenige der Kultur hingegen steht der Gestaltung offen (Hansen 2003: 21).

\subsection{Das Begriffspaar Natur und Kultur}

Zunächst gilt es, die Begriffe der Natur und der Kultur als Ausgangsbereiche der Wirtschaftsmetaphorik inhaltlich zu bestimmen und voneinander abzugrenzen. Unter den Begriff der Natur sollen alle organischen und anorganischen Erscheinungen subsumiert werden, die ohne Zutun des Menschen existieren oder sich entwickelt haben (Duden Universalwörterbuch 2011: 1243). Somit umfasst Natur die Gesamtheit des materiell Vorgefundenen und Phänomene, die sich auch ohne die Existenz des Menschen auf der Erde befinden würden (Hansen 2003: 19).

Demgegenüber bezeichnet Kultur das menschlich Geschaffene und somit Phänomene, die, egal ob materiell oder geistig, auf 
NATUR UND KULTUR ALS BILDSPENDER FÜR DIE KONZEPTUALISIERUNG...

menschliche Urheberschaft zurückführbar sind. „Kultur meint die Veränderung der Natur durch menschliche Tätigkeit, was dazu führt, dass die natürliche Ordnung durch eine vom Menschen geschaffene ersetzt wird." (Hansen 2003: 15). Aus dieser Definition wird deutlich, dass für Kultur menschliche Arbeit vonnöten ist (Hansen 2003: 14). An diesen wertneutralen, wissenschaftlichen Kulturbegriff (Hansen 2003: 16) knüpfen die nachfolgenden Überlegungen an, während die "kognitive Kultur" (Hansen 2003: 237) ausgeklammert wird. Für den Zweck der Beschreibung des Bereichs Wirtschaft, in die der Mensch als Subjekt oder Objekt eingebunden ist, wird das zugrundeliegende Kulturverständnis erweitert um zwei wesentliche Merkmale von Kultur: Kultiviertheit und Zivilisiertheit. Kultiviertheit bedeutet den Höhepunkt der „Veränderung der äußeren und inneren Natur durch Arbeit" (Hansen 2003: 15). Zivilisiertheit meint „die Zähmung der Leidenschaften, oder moderner formuliert, die Disziplinierung der Triebe und Egoismen" (Hansen 2003: 15). Somit besteht der wesentliche Unterschied zwischen dem Naturzustand und dem Raum der Kultur darin, dass Kultur neben Arbeit und Gestaltung auch ein Bewusstsein des eigenen Tuns und Vernunft umfasst, jene Eigenschaften, welche das Tier nicht besitzt (Hansen 2003: 14). Beim Menschen treffen die Bereiche Natur und Kultur zusammen (Hansen 2003: 19):

Einerseits ist der Mensch Naturwesen, welches derselben Kreatürlichkeit unterworfen ist, wie das Tier; andererseits ist er Stifter und Träger der Kultur. (Hansen 2003: 19)

Aus dieser Definition ist zu schließen, dass der Mensch sich sowohl triebhaft verhalten als auch bewusst handeln kann, je nachdem ob er seinem Naturteil oder seinem Kulturteil folgt. Um diesen Aspekt zu vertiefen, wird auf den von Max Weber eingeführten Unterschied zwischen Verhalten und Handeln zurückgegriffen. Ohne zu tief in die komplexe Thematik einzusteigen, soll unter Verhalten eine spontane Reaktion verstanden werden, die ohne große Willensanstrengung und meist ohne Bewusstsein erfolgt (Hansen 2003: 123). Handeln dagegen ist ein menschliches Verhalten (einerlei ob 
Jasmina Mahmutović

äußeres oder innerliches Tun, Unterlassen oder Dulden), das mit einem subjektiven Sinn verbunden ist (Kruse et al. 2012: 91).

Der wesentliche Unterschied zwischen Verhalten und Handeln besteht darin, dass ein Verhalten weder den Verstand noch den Willen noch das Bewusstsein belastet (Hansen 2003: 123). Demgegenüber setzt eine vollwertige Handlung Problembewusstsein voraus, eine Wahl zwischen Zielen und verschiedenen Wegen; sie erfordert eine Phase der Überlegung, die viel Zeit beanspruchen kann, und gipfelt in einem Beschluss (Hansen 2003: 124). Nicht jedes menschliche Handeln ist als soziales Handeln zu begreifen. Unter dem Begriff des „sozialen Handelns“ versteht Max Weber ein Handeln, dass sich am Verhalten anderer orientiert und sich auch auf Werte, Normen und geltendes Recht bezieht (Kruse et al. 2012: 92). Das Massenhandeln oder die bloße Nachahmung fremden Handelns fallen nach Weber nicht unter den Begriff des sozialen Handelns (Kruse et al. 2012: 91). Diese beiden Handlungsarten erfolgen lediglich reaktiv, ohne sinnhafte Orientierung des eigenen an dem fremden Handeln (ebd.).

Konkret geht es in den folgenden Ausführungen nicht nur darum, wirtschaftliche Phänomene zu beschreiben, sondern auch sprachlich gemachte Menschenbilder an der Schnittstelle von Natur und Kultur zu rekonstruieren. Es soll aufgezeigt werden, welche Verhaltensweisen den Akteuren zugeschrieben werden. Werden einzelnen Menschen und Menschengruppen irrationale und instinktgesteuerte, d.h. animalistische Verhaltensweisen zugeschrieben? Oder werden sie als rational und sozial handelnde Menschen dargestellt? Diesen Fragen soll in den folgenden Ausführungen anhand der Metaphernanalyse nachgegangen werden.

\section{Die empirische Untersuchung}

\section{Die Wildnis-Metapher}

Die Wildnis-Metapher schließt an die konventionelle Organismus-Metaphern an, mit der wirtschaftliche Prozess als naturwüchsige und eigendynamische Entwicklungen ausgegeben und damit ebenfalls als dem menschlichen Einfluss entzogen dargestellt wer- 
NATUR UND KULTUR ALS BILDSPENDER FÜR DIE KONZEPTUALISIERUNG...

den (Hebel 1987: 71). Der Aspekt der Naturwüchsigkeit findet in dem analysierten Textmaterial eine kreative Erweiterung in Form der Wildnis-Metapher.

Wildnis dient als Ausgangsbereich für die metaphorische Konzeptualisierung einer Vielzahl von wirtschaftlichen Phänomenen, die dem Laien als fremd, übertrieben und riskant erscheinen. Wildnis-Metaphern verteilen sich im Diskurs auf verschiedene Zielbereiche wie etwa Finanzprodukte, Finanzwirtschaft, Markt und Kapitalismus. Auch die dazugehörigen Akteure werden von der Wildnis-Metapher erfasst, um ihre Handlungen als ungehemmt und gefährlich darzustellen.

Und die ließen sich immer wildere Produkte einfallen, mit immer undurchsichtigeren Namen und immer riskanteren Strukturen. (Der Spiegel 8/2009)

Urwald und Dschungel werden als spezifische Ausprägungen der Wildnis-Metapher auffallend oft für die Konzeptualisierung der Finanzwirtschaft und des Marktes herangezogen, um diese als ein wirres Durcheinander darzustellen. Der Finanzmarkt wird als geschlossener, urwüchsiger und undurchdringlicher Teilbereich der Wirtschaft dargestellt:

Er formuliert so, dass seine Zuhörer sich nicht verirren im

Dschungel der Finanzmärkte. (Der Spiegel 20/2009)

Die Dschungel-Metapher bringt einerseits zum Ausdruck, dass die Finanzwelt dem Laien als undurchschaubar erscheint. Andererseits werden die Bedeutungsaspekte des Unkultivierten und Abgegrenzten auf den Zielbereich übertragen. Der Finanzbereich wird als eine zweite, eine heimliche Welt (Der Spiegel 41/2008) konzeptualisiert, die parallel zur Realwirtschaft existiert.

So richtig unheimlich ist aber der andere Bereich. Das ist der See mit den Krokodilen. Man sieht nichts, der See liegt glatt. Aber im Dunkeln darunter passiert eine Menge. (Der Spiegel 41/2008) 
Jasmina Mahmutović

Die gegenwärtige Wirtschaftslage wird mit „Metaphern der Intrasparenz" (Schwarz-Friesel 2014b) konzeptualisiert, wobei Dunkelheit, trübes Gewässer und Nebel das Bild dominieren. Diese Metaphern sind Ausdruck der Ohnmacht gegenüber einer sich anbahnenden Krise, deren Ausmaß intransparent erscheint.

Das macht die Lage so unheimlich. Es ist wie die Fahrt in einem Nachen auf einem afrikanischen See. Die Besatzung ahnt, dass da Krokodile lauern, aber sie weiß nicht, wie viele und wie groß sie sind. (Der Spiegel 41/2008)

Den Textrezipienten wird der Eindruck vermittelt, dass die wirtschaftlichen Entwicklungen sehr gefährlich sind und zu großen Sorgen und zur Beunruhigung Anlass geben. Gleichermaßen beunruhigend wirkt die Netz-Metapher, mit der unsichtbare Phänomene, wie etwa Globalisierung und Finanzwirtschaft, visualisiert werden.

\section{Die Netz-Metapher}

Abstrakta wie Wirtschaft, Informations- und Kapitalfluss werden als ein Netz oder Geflecht metaphorisiert, das die Welt umspannt und gefangen hält. Die Interdependenzen in der globalen Finanzwirtschaft treten mit dem Ausbruch der Krise wie ein Netz zutage, das scheinbar aus sich selbst heraus die Welt überzogen hat mit Geld- und Kreditströmen.

Unbemerkt haben die Bankenschnösel ein Netz gespannt, haben durch Verkäufe in aller Herren Länder Zusammenhänge geschaffen, unkontrolliert, ohne große Worte. (Der Spiegel 41/2008)

Nun droht dieses undurchsichtige Netz gegenseitiger Zahlungsgarantien zu reißen. (Der Spiegel 43/2008)

Das Netz erscheint nicht als ein menschliches Artefakt, sondern als ein Biofakt und evoziert das Gefühl der Unsicherheit und der 
NATUR UND KULTUR ALS BILDSPENDER FÜR DIE KONZEPTUALISIERUNG...

Verfänglichkeit. Durch die metaphorische Konzeptualisierung der Finanzwelt als Netz wird ein Zusammenhang geschaffen zwischen dem deutschen Sparer und dem US-amerikanischen Kreditnehmer, der zu viel Geld verplempert.

Faktisch hat der Sparer aus Esslingen dem Jim in Nevada sein Häuschen finanziert. (FAS 20/2009)

Das Risiko, dass der deutsche Sparer sein Geld verlieren kann, weil der auf Pump lebende Amerikaner seinen Kredit nicht zurückzahlt, rückt somit in die eigene Lebenswelt.

Weil alles vernetzt ist, wird die Katastrophe gleich zur großen Katastrophe. (Der Spiegel 41/2008)

Zusammenfassend ist festzustellen, dass die Wildnis-Metapher zwei Sorten von Ökonomie entwirft. Die „gute Ökonomie“ ist die Realwirtschaft, die „böse" hingegen die Finanzökonomie (Verdicchio 2006, S. 63).

\section{Die Pflanzen-Metapher}

Die Pflanzen-Metapher ist als spezifische Ausprägung der Wildnis-Metapher im Textmaterial reichlich vorhanden und bezieht sich auf die Gesamtwirtschaft und einzelne Teilbereiche, die scheinbar ganz von allein und ohne menschliches Zutun wachsen, blühen und absterben. Für das Wachstum scheint eine innere Vitalkraft verantwortlich zu sein, keine bewusst handelnden Akteure.

Die Bank war von klein auf groß emporgeschossen und dann in wilden Schüben von groß auf zu groß. (Die Zeit 25/2009)

Die Wall Street erblühte, und aus 5000 Lehman-Leuten wurden 10 000, wurden 15 000. (Der Spiegel 11/2009)

In Großbritannien floriere die Wirtschaft, sagte er. Und das ohne Regeln. (Die Zeit 14/2009) 
Jasmina Mahmutović

Gemeint ist eine Pflanze, die ohne weiteres Zutun gedeiht und Gewinne abwirft, wenn man sie regelmäßig bewässert. Bewachen und pflegen muss man diese Pflanze nicht, denn sie reguliert sich von selbst. Geld erscheint als lebensnotwendige Flüssigkeit bzw. Wasser, das die innere Wachstumskraft der Wirtschaft, der Unternehmen und ökonomischer Kennzahlen stimuliert. Entscheidend ist jedoch das richtige Maß. Zu wenig Geld trocknet die Entitäten aus, zu viel Geld führt zu Degeneration. Im Textkorpus findet die Pflanzen-Metapher eine kreative Erweiterung in Form der Wildwuchs-Metapher. Die Finanzwelt erscheint als ein unkontrolliert wachsender Organismus, der infolge übermäßiger Bewässerung und mangelnder Pflege Auswüchse, Ausschläge, Blasen und Wildwuchs entwickeln konnte.

Weltweit wucherten die Finanzmärkte (...) (Die Zeit 15/2009)

Die Wildwuchsmetapher steht metaphorisch für exzessive ökonomische Entwicklungen, die auf mangelnde staatliche Aufsicht und laxe gesetzliche Regelungen zurückführbar sind.

Angesichts der Niedrigzinspolitik und damit der Verringerung der Risikoprämie ist die Finanzindustrie und ihr Instrumentenkoffer schneller gewachsen als der regulatorische Ordnungsrahmen und dessen Effektivität. (Der Spiegel 19/2009)

Das übermäßige Wachstum in der Wirtschaft wird auf zu viel Fremdfinanzierung zurückgeführt, die den Wirtschaftseinheiten genauso schadet wie Überbewässerung einer Pflanze. Starkes Wachstum wird negativ bewertet, da es kein organisches Wachstum aus eigener finanzieller Kraft, sondern ein durch Schuldenaufnahme gefördertes, ein Wachstum auf Pump, das die Bilanzen aufbläht.

Aus der Wildwuchs-Metapher speisen sich auch unterschiedliche Ausdrücke, mit denen die Finanzialisierung ${ }^{4}$ der Wirtschaft,

4 Die Debatte über die Finanzialisierung benennt verschiedene Veränderungen während der letzten drei Jahrzehnte, die ihren Anfang vorrangig in den USA und Großbritannien nahmen und sich von dort aus verbreitet haben. 
NATUR UND KULTUR ALS BILDSPENDER FÜR DIE KONZEPTUALISIERUNG...

d.h. die Durchdringung des Finanzsektors in die Gesamtwirtschaft und die privaten Haushalte, bildlich dargestellt wird.

Die Gefahr des Kapitalismus liegt in der Ausdehnung des ökonomischen Denkens und des Konkurrenzprinzips auf immer mehr Bereiche unseres Lebens, von den Hochschulen bis hin zu den sozialen Dienstleistungen. (FAS 51/2008)

Kleinanleger, Kirchen, Landkreise und Pensionsfonds hatten dem Wall-Street-Haus ihr Geld anvertraut. (Der Spiegel 11/2009)

Mit Hilfe der Wildwuchs-Metapher wird der Finanzmarkt als ein nicht eingehegter Bereich konzeptualisiert, dessen Ableger in alle anderen Lebensbereiche hinein expandiert sind. Transparent wird hier eines: Die Finanzwelt ist keine abgekoppelte Parallelwelt, sondern eine omnipräsente Kraft, die alle Ebenen und jede Person betrifft. Die Wildwuchs-Metapher steht für die Durchökonomisierung der Gesellschaft. Für das starke Wachstum in der Wirtschaft werden unter anderem die Notenbanken verantwortlich gemacht, die Kapital in die Märkte pumpten und die Märkte mit billigem Geld geflutet haben.

Die Wildwuchs-Metapher liefert nicht nur eine plausible Erklärung für die Fehlentwicklungen in der Wirtschaft, sondern legt auch bestimmte Handlungen bzw. Gegenmaßnahmen nahe. Es kommen diverse Regulierungs-Metaphern aus den Bereichen Landwirtschaft und Forstwirtschaft zum Einsatz, mit denen die Naturwüchsigkeit der Wirtschaft kultiviert werden soll.

Die internationale Gemeinschaft soll den Wildwuchs regulieren (Der Spiegel 6/2009)

Dazu gehören z.B. die Deregulierung und Öffnung vormals national segmentierter Finanzmärkte, die verstärkte Vermarktlichung von Finanzbeziehungen, die explosionsartige Verbreitung neuer Finanzinstrumente, sowie die dramatische Ausweitung des Kredit- und Anlagegeschäftes für Privatkunden in Form vom Hypotheken, Konsumentenkrediten und der privaten $\mathrm{Al}$ terssicherung (Stockhammer 2008: 184, zit. nach (Heires et al. 2011: 38). 
Jasmina Mahmutović

Die Wall Street hat jetzt eine Schrumpfkur vor sich, ein brutales Zurechtstutzen der außer Rand und Band geratenen Branche. (Die Zeit 39/2008)

Finanzminister Peer Steinbrück will die Bank nun zurechtstutzen. (Der Spiegel 39/2008)

Die Steueroasen sollen ausgetrocknet und die Hedgefonds an die Leine gelegt werden. (Die Zeit 47/2008)

Die zitierten Belege enthalten eine unscheinbare Perspektivierung des Finanzministers als Förster. Geldpolitische Maßnahmen, wie etwa Verstaatlichung von Banken, Verfolgung von Steuerflüchtigen und Verschärfung von Gesetzen, gelten in diesem Metaphernbereich als Kultivierung.

Ein Schlüsselwort in dem analysierten Textkorpus ist das aus der Forstwirtschaft stammende Konzept der Nachhaltigkeit. So wird für eine Abkehr von kurzfristigen Gewinnen und eine Zuwendung zu nachhaltigen Erfolgen plädiert, wie folgender Beleg exemplarisch zeigt:

Nachhaltigkeit hat für uns deshalb erste Priorität und steht ganz eindeutig vor kurzfristiger Gewinnoptimierung. (Der Spiegel 47/2008)

Zusammenfassend ist für die Pflanzen-Metapher festzuhalten, dass Wirtschaft überwiegend über den Bereich Natur als Zustand der Unkultiviertheit konzeptualisiert wird. Es zeigt sich, dass ein neoliberalistisch geprägtes Wirtschaftsgefüge in Deutschland keine Zukunftsberechtigung hat, und dass sich der Staat aus dem Wirtschaftsleben nicht heraushalten kann. Dafür steht exemplarisch folgender Beleg:

Die Wirtschaft brauche »die ordnende Hand der Staaten, die Regeln setzen und auch durchsetzen«. (Die Zeit 50/2008) 
NATUR UND KULTUR ALS BILDSPENDER FÜR DIE KONZEPTUALISIERUNG...

Mit diversen Kultivierungs-Metaphern wird nahegelegt, dass dem Staat eine wichtige Rolle bei der Gestaltung und Lenkung der Wirtschaft zukommt.

\section{Die Tier-Metapher}

Wurden bis hierher Metaphernkonzepte aus dem Bereich Flora analysiert, wendet sich dieses Kapitel der Tier-Metapher zu. Die Tier-Metapher ist in dem Textkorpus ebenfalls keine Neuheit 5 . Neu sind jedoch die kreativen Erweiterungen dieses Metaphernbereichs, mit denen bisher unbekannte Bedeutungsaspekte beleuchtet werden, wie auch neuartige, bisher unvernetzte Konzeptkombinationen hinzutreten.

Im Folgenden soll zunächst gezeigt werden, wie die Akteure über den Rückgriff auf diverse Tier-Metaphern abgewertet und stigmatisiert werden. Im Anschluss daran soll skizziert werden, wie wirtschaftliche Prozesse anhand von Metaphern in das Tierreich versetzt werden sowie welche Handlungen jeweils nahegelegt werden.

Die Assoziation mit Tieren ist eine der effektivsten sprachlichen Strategien, Menschen abzuwerten. Paul zufolge (2004: 311), spricht der Tierbegriff nicht notwendig von "konkreten Tieren“, sondern meint ein abstraktes „Tier-Konstrukt", eine stereotype, zoologische Konzeption vom Tier. Tier-Konstrukte repräsentieren nicht die Bezugsobjekte an sich, sondern nur unsere Vorstellungen von ihnen.

Mit Tiermetaphern wird ganzen Menschengruppen insgesamt das Menschsein abgesprochen, indem man feststellt, „einer solchen Gruppe fehle es an einem (oder mehr als einem) wesentlichen Merkmal, das den Menschen »eigentlich « erst zum Menschen mache" (Hermanns 2012b: 43). Ein wesentliches Merkmal, das den Menschen vom Tier unterscheidet, so hat sich gezeigt, ist die Disziplinierung der Triebe und Egoismen (Hansen 2003: 15). Die Analyse des Textmaterials zeigt, dass insbesondere den Akteuren in der Wirtschaft das „Menschsein wegargumentiert" (Hermanns

$5 \quad$ Zur Tiermetaphorik in der Wirtschaftssprache siehe u. a. (Musolff 1991), (Baldauf 1997) (Jäkel 2003). 
Jasmina Mahmutović

2012b: 43) wird, indem sie entweder explizit als Tiere nominiert werden oder indem ihre Handlungen auf die animalisch gedeutete Triebebene herabgestuft werden. Folgende Belege illustrieren, wie Menschengruppen als von Affekten, Trieben und Egoismen gesteuerte Tiere konzeptualisiert werden:

....,wie Schweine am Trog“ verhielten sich Anleger am Markt. (Der Spiegel 20/2009)

In einem Boom, in dem die animalischen Triebe ("animal spirits") anstelle rationaler Überlegung treten, gilt das nicht unbedingt. (FAS 30/2009).

Rückwirkend betrachtet sieht manches in unserer Branche gierig aus, selbstbezogen und realitätsfremd, als ginge die Gesellschaft drum herum sie gar nichts an. (Der Spiegel 19/2009)

Anders als beim Tier, dessen instinktgesteuerter Trieb auf die Befriedigung oft lebensnotwendiger Bedürfnisse zielt, ist der Trieb bestimmter Diskursakteure auf die Maximierung von Gewinnen ausgerichtet.

Der Trieb, Reichtum zu erwerben, steckt in uns drin. Wenn dieser Trieb aber den Gesetzen des Urwaldes überlassen wird, nach denen der Stärkste sich gnadenlos durchbeißt, dann ist das nicht sozial. (FAS 18/2009)

Die Tier-Metapher findet eine spezifische Ausprägung in der im Korpus stark belegten Herdentrieb-Metapher. Dafür steht exemplarisch folgender Beleg:

Sein Kollege Robert Shiller, Professor an der Yale-Universität, hat bei Keynes gefunden, was für ihn das Ausmaß dieser Systemkrise erklärt: den „animal spirit“ im Kapitalismus, den Herdentrieb in einem System, das auf ganz und gar unmathematischen Wegen nach Geld strebt, unberechenbar, irrational. (Der Spiegel 20/2009) 
NATUR UND KULTUR ALS BILDSPENDER FÜR DIE KONZEPTUALISIERUNG...

Mit der Herdentrieb-Metapher wird den Wirtschaftsakteuren reflexives und nicht-soziales Handeln zugeschrieben. Nachahmung fremden Handelns und Massenhandeln ist - wie bereits erwähnt - kein soziales Handeln. Die den Akteuren zugeschriebenen Eigenschaften Irrationalität und Massenhandeln kommen in den Bezeichnungen Herde, Horde, Schar, Rudel etc. zum Ausdruck. Die Akteure erscheinen als unselbständig denkende und handelnde Menschen, die sich willenlos führen oder treiben lassen. Zentrale sind dabei die Konzepte Ratten und Lemminge und die vermeintlichen Verhaltensweisen dieser Tiere. Folgende Ausdrücke sind belegt: rat-race, Rattenrennen um Gewinnmargen, Rattenrennen um Rendite, Wettlauf der Lemminge etc.

Verhalten sich alle gleich .... wie die Lemminge (Der Spiegel 1/2009)

Mit der Lemming-Metapher wird stereotypisches Wissen über diese Tierart auf die Wirtschaftsakteure übertragen. Dazu gehört auch das Wissen über den Mythos, Lemminge würden kollektiven Massenselbstmord begehen, indem sie sich ins Meer stürzen ${ }^{6}$. Der damalige Finanzminister Steinbrück greift auf diesen Mythos zurück und koppelt sie an die von ihm in Umlauf gebrachte Abgrund-Metapher, um den Laien die Ursachen für die Krise zu erklären:

Auf 4,5 Billionen Dollar schätzt der Internationale Währungsfonds die Vernichtung von Finanzvermögen in der Welt. Es ging zu wie in einem Rennen von Lemmingen: Alle liefen mit, und viele liefen in den Abgrund. (FAS 37/2009)

Die Lemming-Metapher lässt sich in folgende Proposition auflösen: Wirtschaftsakteure sind ein selbstmordgefährdete Gruppe. Explizit kommt die Selbstmordgefährdung in folgenden Beleg zum Ausdruck:

$6 \quad$ Das Mythos wurde mit dem US-amerikanischen Tierfilm Weiße Wildnis aus dem Jahr 1958 verbreitet und findet sich in diversen Computerspielen wieder. 
Jasmina Mahmutović

Die Wirtschaftselite kurzsichtig, gierig, selbstmordgefährdet;

(Der Spiegel 20/2009)

Festzuhalten bleibt, dass mit der Herdentrieb-Metapher die unterstellte Gemeinsamkeit ,Massenhandeln' mit allen Implikationen in den Bereich des Vorbewussten und Irrationalen verschoben wird. Dieser Bedeutungsaspekt wird durch die Ausdrücke das haben alle gemacht, mitgespielt, wollte dabei sein verstärkt.

Alle haben mitgemacht, nicht nur die Banken.

(Der Spiegel 1/2009)

Die Tatsache, dass Massen manipulierbar und verführbar sind, kommt in den folgenden Belegen zum Ausdruck:

„Wir haben uns blenden lassen«, sagt ein hochrangiger Beamter. (Die Zeit 15/2009)

Es war ja nicht nur die Industrie, die verführte. Die Menschen wollten sich auch verführen lassen. (Der Spiegel 1/2009)

Die Mitspiel-Metapher mutet seltsam vertraut an. Mit ihr wird das Bild eines von einer Minderheit verführten Kollektivs etabliert, in dem alle nur mitgemacht haben und sich am Ende keiner Schuld bewusst sind. Man sei mitgefangen, mitgehangen und müsse nun „kollektive Demut“ entwickeln. (Der Spiegel 19/2009)

Stand bei der Lemming-Metapher das massenbedingte, reaktiv verursachte Handeln im Vordergrund, so dominiert bei der Rudel-Metapher die rücksichtslose Absicht, aus der Wehrlosigkeit des Opfers einen Vorteil zu ziehen.

Oft im Rudel prügeln Fonds die Kurse angeschlagener Firmen in den Boden, um so ihre Profite zu maximieren. (Der Spiegel 39/2009) 
NATUR UND KULTUR ALS BILDSPENDER FÜR DIE KONZEPTUALISIERUNG...

Die Rücksichtslosigkeit der Hedge-Fonds und internationaler Investoren wird auch mit der negativ belasteten Heuschrecken-Metapher zum Ausdruck gebracht.

Das Geldhaus hatte die Forderungen aus Piper größtenteils weitergereicht - an fünf Hedgefonds aus Übersee, Heuschrecken wie Caner, nur größer und aggressiver. (Der Spiegel 25/2009)

Die Hedgefonds haben sich darauf spezialisiert, das noch vorhandene Fleisch vom Knochen sogenannter Distressed Investments zu schälen. (Der Spiegel 25/2009)

Die Heuschrecken-Metapher ist im Anschluss an die sogenannte Kapitalismusdebatte ${ }^{7}$ zu einem zentralen Bestandteil des öffentlichen Diskurses geworden (Ziem 2008b: 115). Ziem zeigt in zwei detaillierten inhaltlichen Analysen (2008a: 367-440; 2008b), dass sich metaphorische Verwendungsweisen von Heuschrecke zwar schon in der Bibel zur Konzeptualisierung der Strafe Gottes finden, dass aber der heutige Gebrauch eher für das kapitalistische Prinzip der Profitmaximierung (Ziem 2008b: 114) steht. Ein Teil der aktuellen Bedeutung der Heuschrecken-Metapher markiert eine gewisse geschichtliche Kontinuität der sozialen Stigmatisierung von Menschengruppen, die als fremde Eindringliche empfunden werden (Ziem 2008b: 118).

Die Heuschrecken-Metapher im Rahmen der Kapitalismus-Debatte als Form der Kapitalismuskritik einzusetzen (...), schließt unmittelbar an die Stigmatisierung von Juden als raffgierige Kapitalisten an, zumal meist angloamerikanische Finanzinvestoren in der Kritik stehen und standen. Der gegenwärtige Trend geht zudem dahin, Heuschrecke als Stigmawort im Schnittfeld von Antikapita-

7 In einem Interview für die Bild am Sonntag hat der damalige Bundesarbeitsminister Franz Müntefering die Metapher zum ersten Mal benutzt. Da heißt es: „Manche Finanzinvestoren verschwenden keine Gedanken an die Menschen, deren Arbeitsplätze sie vernichten. Sie bleiben anonym, haben kein Gesicht, fallen wie Heuschreckenschwärme über Unternehmen her, grasen sie ab und ziehen weiter." (Ziem 2013: 230) 
lismus und Antiamerikanismus zu etablieren. (Ziem 2008b: 118).

Die Heuschrecken-Metapher scheint auch im Diskursverlauf immer wieder auf; etwa um Kritik an Investoren und Hedge-Fonds aus den USA und Großbritannien sowie an den Wirtschaftsgefügen dieser Länder zu üben. Großbritannien wird z. B. als Land der Heuschrecken (Der Spiegel 25/2009) bezeichnet, während die USA als Mutterland des ungezügelten Kapitalismus (Der Spiegel 8/2009) oder Land des Raubtierkapitalismus (Der Spiegel 20/2009) nominiert werden.

Die lexikalisierte Metapher „Raubtierkapitalismus“ steht laut Duden-Online für eine „ohne Rücksicht auf andere Belange nur nach möglichst großem Profit strebende Form des Kapitalismus" ${ }^{\text {“8 }}$. Die Raubtier-Metapher wird im Textkorpus kreativ verknüpft mit einer Fülle von Entfesselungs-Metaphern, mit denen die neoliberalistische Ausgestaltung des Kapitalismus und die damit einhergehende Deregulierung, d.h. Verringerung staatlicher Eingriffe in das Marktgeschehen, als Entfesselung des Kapitalismus (Der Spiegel 44/2008) konzeptualisiert werden. Die Angelsachsen hätten das Tier entfesselt und nicht ausreichend überwacht, so konnte es aus seinem Mutterland, den USA, ausbrechen und auf den Rest der Welt übergreifen.

Der entfesselte Finanzkapitalismus ist die Spätfolge einer politischen Schule, die sich in der Nach-Thatcher- und Nach-Reagan-Zeit in vielen Ländern etabliert hat. (FAS 37/2009)

Regierungen haben in den letzten drei Jahrzehnten die Märkte von Fesseln befreit, zunächst in den USA und in Großbritannien, später auch in Deutschland. (Der Spiegel 20/2009)

Das Bild wird verstärkt durch Metaphern der Zügellosigkeit, die versprachlicht werden als hemmungslos, enthemmt, unkontrolliert, wild, zügellos, ungezügelt, exzessiv, maßlos.

8 https://www.duden.de/suchen/dudenonline/Raubtierkapitalismus (Abrufdatum 20.10.2017) 
NATUR UND KULTUR ALS BILDSPENDER FÜR DIE KONZEPTUALISIERUNG...

Die gleichermaßen komplexen wie abstrakten Phänomene Markt und Kapitalismus werden in diesen wie ähnlichen Äußerungen als entfesseltes Raubtier konzeptualisiert. Die Entfesselungs-Metapher legt auch entsprechende Handlungen nahe. Mit dem Auftreten der Akteure - Wirtschaftsexperten und Vertreter der Politik - werden die von ihnen favorisierten Maßnahmen der Domestizierung diskutiert. Wirtschaftspolitische Maßnahmen werden zu Zähmungen und Fesselungen, um den Kapitalismus einzufangen, zu beruhigen und an die Leine zu legen, wie folgende Belege zeigen:

Seit 1989/90 gibt es kein Ausbrechen mehr aus dem Universum des Kapitalismus - es kann nur um eine Zivilisierung und Zähmung der kapitalistischen Dynamik von innen gehen. (Die Zeit 46/2008)

Nicht, weil die Bürger den Kapitalismus satthätten, sie wollen ihn lediglich zügeln. (Der Spiegel 15/2009)

Als Bundesfinanzminister Peer Steinbrück vor zwei Jahren Hedgefonds an die Leine nehmen wollte, war Brown dagegen. (Die Zeit 14/2009)

Deutschland würde die Banken weitaus stärker an die Kandare nehmen als die USA oder Großbritannien. (Die Zeit 11/2009)

Zusammenfassend ist festzuhalten, dass die Wirtschaft anhand der Tier-Metapher als ein wilder und somit äußerst bedrohlicher Bereich dargestellt wird, der ohne den Einfluss des Staates animalische Züge annehmen kann, wie folgender Beleg zeigt:

Der entfesselte Markt muss reguliert und kontrolliert werden, gewiss. (Die Zeit 47/2008)

Mit Metaphern der Zähmung, Zügelung und Zivilisierung wird nahegelegt, dass der barbarische Naturzustand durch mensch- 
Jasmina Mahmutović

lichen Eingriff in einen Kulturzustand überführt werden muss. Die Darwinistische Metapher

Unter Bezugnahme auf das darwinistische Bild vom Daseinskamp als „survival of the fittest“ werden die Abläufe und Akteure in der Wirtschaft in den Wissensrahmen der Evolutionstheorie eingebettet, wo sich der Stärkere durchsetzt und der Schwache nur geringe Überlebenschancen hat (vgl. Musolff 1991, Jäkel 2003).

Der Stärkere, der sich in der freien Wildbahn der Konkurrenzgesellschaft durchsetzt, (Die Zeit 46/2009)

Der Markt wird als ein Territorium konzeptualisiert, in dem Unternehmen in einem gegenseitigen evolutionären Kampf ums Überleben stehen. Dabei gilt folgender Grundsatz:

Töte den Feind, bevor der Feind dich tötet. (Der Spiegel 11/2009)

Der Markt erscheint als eine begrenzte Ressource, um deren Anteile hart gekämpft wird. So erscheint es folgerichtig, dass die Stärksten und Größten diesen Darwin'schen Selektionskampf überleben, indem sie die Schwachen fressen, schlucken und verdauen. Gelegentlich werden finanziell schwache und untaugliche Unternehmen in einem gnadenlosen Vorgang natürlicher Selektion vom Markt selbst ausgemerzt und weggefegt. Diese Rolle wird in dem analysierten Textkorpus von der Wirtschaftskrise übernommen:

Es muss vorher in der Wirtschaft starke Übertreibungen gegeben haben. Dann kann eine Krise - ohne Staatseingriff - eine reinigende Wirkung haben, weil fehlgeleitete Unternehmen vom Markt verschwinden. (FAS 49/2008)

Die Auswertung des Textmaterials führte zu dem Ergebnis, dass die Darwinistische Metapher eine kreative Erweiterung erfahren hat, indem der Daseinskampf und der Selektionsprozess auf 
NATUR UND KULTUR ALS BILDSPENDER FÜR DIE KONZEPTUALISIERUNG...

zwei miteinander konkurrierende Wirtschaftsformen ausgeweitet werden. So kämpft die soziale Marktwirtschaft mit dem angelsächsischen Kapitalismus um eine Existenzberechtigung auf dem deutschen Markt. Die Vertreter der Politik unterstützen diesen Kampf, wie folgende Belege zeigen:

Es kann nicht sein, dass wir die Kapitalisten einfach machen lassen. Man muss Regeln finden, um die globale Finanzindustrie an die Kette zu legen. (Die Zeit 44/2009)

Aber wenn wir den Raum den Stärksten überlassen, weil sie das meiste Geld haben, die dicksten Muskeln, geht das nicht gut. (Die Zeit 44/2009)

Das metaphorische Szenario von Wirtschaft als Kampf ums Überleben der Stärksten lässt sich wie folgt zusammenfassen: Unternehmen und Wirtschaftsformen werden animiert als Rivalen, die in einem scharfen Kampf ums Überleben miteinander konkurrieren. Sie treten als Tiere auf, durch deren Beteiligung am Markt der Darwin'sche Kampf mit seiner natürlichen Selektion zum Kampf um Marktanteile, Kunden und Renditen eskaliert. Als naturgegeben sind Unternehmen unterschiedlich fit und nur die fittesten überleben den Kampf ums Dasein. Die natürliche Auslese wird vom Markt unterstützt, der schwache Unternehmen eliminiert und Lebensraum für die starken schafft.

\section{Die Jagd-Metapher}

Jagdbezogene Metaphern bilden einen wohletablierten Metaphernbereich der Wirtschaftssprache (Musolff, 1991) und lassen sich auch im Textkorpus an einer Fülle von Belegen nachweisen. Die Jagd als anschauliche Form des Nahrungserwerbs durch Rückgriff auf in der Natur vorhandene, wahrnehmbare Lebewesen dient als Ausgangsbereich für die kognitive Bewältigung von komplexen wirtschaftlichen Abläufen aus dem abstrakten tertiären Sektor. Spezifische wirtschaftsthematische Bereiche, in denen Jagd- 
Jasmina Mahmutović

metaphorik in dem analysierten Textmaterial häufig zum Einsatz kommt, sind ökonomischer Wettbewerb, Finanzdienstleistungen und Krisenmanagement. In allen drei Bereichen knüpft die Jagdmetaphorik mehr oder weniger an die darwinistische Sicht des Wirtschaftsgeschehens an. Um aufzuzeigen, welche Aspekte der Jagd bei der Metaphorisierung von wirtschaftlichen Geschehnissen relevant sind, soll zunächst der Bilderspenderbereich Jagd skizziert werden. In einem zweiten Schritt werden die einzelnen Ausprägungen der Jagd-Metapher näher beschrieben.

Die Jagd ist entweder eine Freizeitbeschäftigung oder eine Wirtschaftsform. In beiden Fällen wird auf Vorgefundenes zurückgegriffen. Dient die Jagd dem Nahrungserwerb, so handelt es sich um eine Tätigkeit, die der Mensch mit dem Raubtier teilt (Hansen 2003: 14). Die Jagd als Ernährungsform wird heute mit Naturvölkern in Zusammenhang gebracht, als eine prä-staatliche oder staatenlose Wirtschaftsform. Wesentliche Merkmale der Jagd sind die Nicht-Sesshaftigkeit der Jäger, die Kurzfristigkeit der Nahrungssicherung sowie die starke Abhängigkeit vom Zufall. Diese Ernährungsform erfordert wenig Arbeit, liefert aber eine gehaltvolle Nahrung. Jagd erfordert Körperkraft, Schnelligkeit und Geschick. Es handelt sich um eine Männerdomäne, denn Jagd impliziert Maskulinität, eine zielgerichtete, körperliche Tätigkeit, ein aktives Eingreifen und unter Kontrolle bringen von Gegenständen des Interesses (Hansen 2003: 134).

Bei der Konzeptualisierung der Wirtschaft als Jagd werden die Akteure in Angreifer und Opfer eingeteilt. Die Angreifer sind im Falle der Finanzwirtschaft die Investmentbanker, Trader und Hedge-Fonds-Manager. Die Auswertung der Nominationen und Prädikationen für diese Personengruppe zeigte, dass das Investmentbanking als typische Männerdomäne dargestellt wird. Die Akteure sind überwiegend junge, starke, schnelle, aggressive Kerle, Jungs, Kids ${ }^{9}$ und besitzen somit die Eigenschaften, die für eine erfolgreiche Jagd erforderlich sind. Die Anleger hingegen werden nominiert als Opfer, ältere Personen, Rentner, ahnungslose Dritte und typisiert

$9 \quad$ Als typische Attribuierungen erscheinen stark, flink, aggressiv, hungrig, schnell, egoistisch, rücksichtslos, gierig und raffgierig. 
NATUR UND KULTUR ALS BILDSPENDER FÜR DIE KONZEPTUALISIERUNG...

als alt, doof, naiv, nichtsahnend, ahnungslos. In dieser dualistischen Beschreibungsweise erscheinen Anleger als eine ideale Jagdbeute. Die Jagd-Metapher bringt zum Ausdruck, dass in der Finanzwirtschaft nicht produziert, sondern auf Vorgefundenes zurückgegriffen wird: das Geld anderer Leute. Als Köder und Lockmittel werden diverse Finanzprodukte eingesetzt; den Anlegern schmackhaft gemacht und auf den Markt geworfen. Das Gespräch mit dem Kunden, in dem die zweifelhaften Produkte verkauft werden, wird als Falle konzeptualisiert. Dazu folgender Beleg:

„Aber da“, sagt Launhardt heute, „schnappte die Falle schon zu." (Der Spiegel 3/2009)

Der aktive Zugang auf die Kundschaft wird als Kundenfang, Jagd oder Treibjagd versprachlicht.

Viele Banker ärgert es derzeit, wenn ausgerechnet die teilverstaatlichte Commerzbank mit besonders üppigen Zinsen und aggressiver Werbung auf Kundenfang geht. (Der Spiegel 9/2009)

Werden Börsenhändler und Hedge-Fonds-Manager als Angreifer konzeptualisiert, dann erscheinen sie als besonders aggressiv und skrupellos.

Dann folgte am Donnerstag das große Halali auf Morgan Stanley, die neben Goldman Sachs letzte große überlebende Investmentbank. (Der Spiegel 39/2008)

Hier haben die Händler längst ihre Treibjagd auf das nächste Opfer eröffnet. (Der Spiegel 39/2008)

Aufgrund der Finanzkrise attackierten die Spekulanten die Ostwährungen wie etwa den ungarischen Forint nur vorsichtig. „Die Fonds haben derzeit nicht die Kraft für eine großangelegte Spekulationsattacke" (Der Spiegel 46/2008) 
Bei dem Begriff Spekulation ${ }^{10}$ handelt es sich um eine Übertragung aus dem Jagdbereich in die Wirtschaftssprache. Auch wenn dieser metaphorische Ursprung erblasst ist, zeigt diese Konzeptkombination, dass die Wahrnehmung der Wirtschaft als Jagd keine Erfindung unserer Zeit ist, sondern schon seit dem 18. Jahrhundert belegt ist.

In dem metaphorischen Szenario „Finanzwirtschaft ist Jagd“ stehen die Anleger metonymisch für ihr Geld, das als Beute konzeptualisiert wird. Als Beutefleisch erscheinen aber auch Deals, Margen, Gewinnmargen, Renditen, Gewinne, Erträge, Dividenden, Boni und Abfindungen, die mit den Attributen saftig, dick und fett versehen werden.

Gerade aber das Investmentbanking hat in einigen Instituten schon wieder zu saftigen Gewinnen geführt. (FAS 23/2009)

Und was ist mit der zügellosen Jagd nach Rendite? (FAS 8/2009)

Bei der Jagd auf Kunden werden diverse Wertpapiere, üppige Zinsen und verlockende Angebote als Köder eingesetzt. Bei der Köder-Metapher schwingt ein wesentlicher Bedeutungsaspekte mit, der auf die Finanzprodukte übertragen wird: Ein Köder ist nicht immer etwas Essbares, sondern er kann Nahrung auch vortäuschen oder Gift enthalten. In dem analysierten Textmaterial ist die Giftköder-Metapher implizit präsent und lässt sich an den adjektivischen Attributen für Wertpapiere ablesen: verseucht, giftig, faul, gefährlich, riskant. Solche Wertpapiere wurden kunstvoll verpackt und weiterverkauft.

So konnte Credit Suisse aus einer ganzen Reihe solcher Immobilienkredite Pakete schnüren, um sie dann mit anderen Finan-

10 Eine Entlehnung aus dem lat. speculārī '(umher)spähen, sich umsehen, auskundschaften, beobachten', die seit dem 18. Jahrhundert in der Kaufmannssprache in der Bedeutung 'kaufmännische Berechnung, riskante, auf Gewinn zielende Unternehmung' „Spekulation“ belegt ist. Bereitgestellt durch das Digitale Wörterbuch der deutschen Sprache, <https://www.dwds.de/ wb/Spekulation>, abgerufen am 20.10.2017. 
NATUR UND KULTUR ALS BILDSPENDER FÜR DIE KONZEPTUALISIERUNG...

zierungen zu hochverzinsten, aber riskanten Wertpapieren auf den Markt zu werfen. (Der Spiegel 25/2009)

Mit der Giftköder-Metapher wird eine moralisch verwerfliche Art des Wirtschaftens dargestellt. Sie impliziert, dass schlechte Kredite bewusst zu Paketen gebündelt und weiterverkauft wurden, obwohl bekannt war, dass sie giftig sind und Vermögen vernichten können. Folgender Beleg zeigt, dass dieser Trend aus den USA kommt:

Die USA sind heute eine Volkswirtschaft, die das Ersparte anderer Völker ansaugt. (Der Spiegel 18/2009)

Mit der Jagd-Metapher wird die globalisierte Welt in Reviere eingeteilt. Internationale Banken mit Filialen in Deutschland werden als Wilderer metaphorisiert und somit als Eindringliche empfunden. Der Handel mit zweifelhaften Wertpapieren wird als eine dem deutschen Bankengeschäft fremde Tätigkeit dargestellt.

Das große Geschäft aber, so stellten die Banken fest, macht man ohnehin nicht mehr mit Sparern und Kleinkrediten, sondern mit Wertpapiergeschäften und Firmenübernahmen. Internationale Großbanken bewiesen das und wilderten auch im deutschen Revier. (FAS 41/2008)

Mit der Konzeptualisierung von Wirtschaft als Jagd wird auch eine Kritik an den angelsächsischen Geschäftsmodellen angestoßen.

Den Crash 08 hätten allein die raubtierkapitalistischen Angelsachsen zu verantworten (Die Zeit 32/2009)

Die Jagd der Angelsachsen nach kurzfristiger Rendite und immer höheren Quartalsergebnissen sei ursächlich für die Krise, die sich nun wie ein Ölteppich ausbreite, analysierte Steinbrück. (FAS 38/2009)

Zusammenfassend lässt sich für die Jagd-Metapher folgendes 
Jasmina Mahmutović

festhalten: Die Wirtschaft und ihre Akteure werden in einem Urzustand dargestellt, in dem der Mensch den Raum der Kultur und der langfristigen Nahrungssicherung durch landwirtschaftliche Tätigkeit noch nicht betreten hat. Es dominieren Bilder der Kurzfristigkeit, der schnellen Gewinnanhäufung und der Rücksichtslosigkeit auf Andere.

\section{Das Wirtschaftsgefüge - Tier oder Mensch?}

Im Folgenden wird die in dem Textmaterial deutlich gewordene Kapitalismuskritik aufgegriffen. Es soll aufgezeigt werden, welche Metaphern, neben den bereits besprochenen, jeweils für die Konzeptualisierung des angelsächsischen Kapitalismus und der deutschen Marktwirtschaft gebraucht werden. Im Rahmen einer Nominationsanalyse soll dargestellt werden, mit welchen Nominalphrasen und Prädikationen auf den Kapitalismus und auf die Marktwirtschaft Bezug genommen wird. Für den Kapitalismus ergibt sich folgendes Bild:

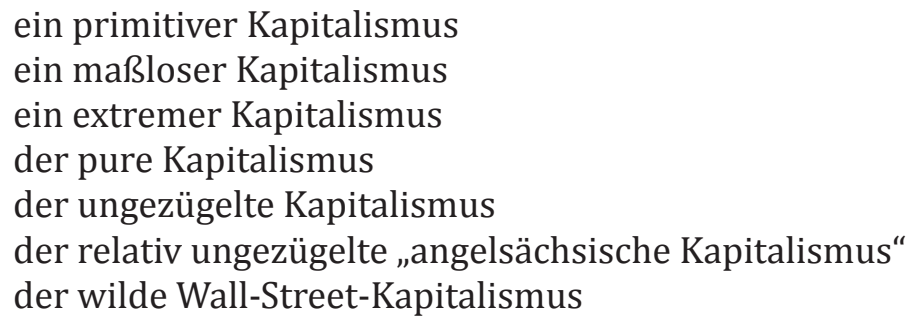

Die Vielzahl der unterschiedlichen adjektivischen Attribuierungen zeigt, dass es nicht den einen Kapitalismus gibt. Vielmehr existieren für die Akteure eine große Anzahl unterschiedlicher Kapitalismusformen, die überwiegend negativ bewertet werden.

Als Prädikate werden dem Nomen Kapitalismus zugeschrieben: unberechenbar, irrational, rücksichtlos, zügellos, enthemmt, unkontrolliert, unreguliert, menschenfeindlich.

Die attributiv und prädikativ gebrauchten Adjektive haben eine stark negativ wertende Bedeutung und rufen eine ablehnende Haltung hervor. Der Kapitalismus wird als etwas Andersartiges, dem deutschen Wirtschaftsverständnis Fremdes, wahrgenommen. 
NATUR UND KULTUR ALS BILDSPENDER FÜR DIE KONZEPTUALISIERUNG...

Es wird plädierten für einen netten, neuen, aufgeklärten, eingehegten Kapitalismus.

Es hat sich aber weltweit ein anderer Kapitalismus entwickelt, der exzessiv wurde und keine soziale Komponente mehr kannte. (FAS 18/2009)

Bei der Analyse der deutschen Wirtschaftsordnung fällt auf, dass diese wahlweise als Kapitalismus oder Marktwirtschaft bezeichnet wird. Daher war der Kontext, in den Kapitalismus eingebettet ist, maßgeblich bei der Eruierung der Analyseeinheiten. Es ergab sich folgendes Bild:

die freie Marktwirtschaft

die soziale Marktwirtschaft

eine vernünftige, funktionierende soziale Marktwirtschaft

eine gestaltete Marktwirtschaft

der rheinische Kapitalismus

ein Kapitalismus mit menschlichem Antlitz

Ordnung

Modell

unser Modell

Als Prädikate treten auf: ist ein Zivilisationsprodukt, ist das beste Modell etc.

Die Nominations- und Prädikationsanalyse zeigt, dass die deutsche Wirtschaftsform überwiegend positiv bewertet wird, indem auf die soziale und menschliche Komponente des deutschen Modells verwiesen wird. Der Begriff der „sozialen Marktwirtschaft“ wird als „Fahnenwort für die bundesrepublikanische Wirtschaftsordnung" gebraucht. (Stötzl et al. 1995: 49) und von den Wirtschaftsformen anderer Länder abgegrenzt.

Mit Bezug auf die zentrale Fragestellung dieser Arbeit, ob die Wirtschaft in den Bereich Natur oder Kultur fällt, lässt sich festhalten: Aus der Analyse der Natur- und Kulturmetaphorik ergibt sich, dass neoliberalistische Wirtschaftsgefüge eindeutig in den Bereich 
Jasmina Mahmutović

der Natur fallen. Sie werden als natürliche Systeme und Tiere dargestellt, die ohne menschlichen Eingriff zu funktionieren scheinen. Die deutsche Wirtschaftsform fällt in den Bereich der Kultur. Die Marktwirtschaft wird entweder als ein Mensch oder als eine vom Menschen geschaffene und daher modellierbare Wirtschaftsordnung dargestellt. Folgender Beleg aus dem Textkorpus soll das illustrieren:

Es geht um die Frage nach der Wirtschaftsordnung, in der die Bürger künftig leben wollen. Ein System, in dem eher die unsichtbare Hand des Marktes nach den Gesetzen von Angebot und Nachfrage regiert, wie es schon der Moralphilosoph und Ökonom Adam Smith einst forderte. Oder eine Ordnung, in der die sichtbare Hand des Staates die Wirtschaft maßgeblich lenkt. (Der Spiegel 9/2009)

\section{Zusammenfassung der Analyseergebnisse}

Anhand des Metapherngebrauchs in dem analysierten Textmaterial wurde gezeigt, dass das abstrakte Phänomen Wirtschaft auf unterschiedliche Weise konzeptualisiert wird. Die am häufig eingesetzten Metaphern stammen aus den Bereichen Wildnis, Dschungel, Wildwuchs, Raubtiere, Darwinistischer Kampf ums Dasein und Jagd. Unterschiedliche Konzeptualisierungen sind möglich, weil beim Metapherngebrauch nur einzelne Aspekte des Ausgangsbereichs auf den Zielbereich übertragen und beleuchtet werden, während alle anderen Aspekte im toten Winkel der Metapher bleiben. Für die Wirtschaft im Spannungsfeld von Natur und Kultur ergab sich, dass der gegenwärtige Zustand überwiegend mit Metaphern aus dem Bereich der Natur bzw. Wildnis dargestellt wird. Wirtschaftliche Prozesse werden durch den Gebrauch als naturgegeben und vom Menschen nicht beinflussbar dargestellt. Die Wirtschaftsakteure werden entweder als Jäger oder Wilderer, die den Raum der Kultur noch nicht betreten haben, dargestellt, oder aber zu Tieren herabgestuft. Es dominiert das Bild der kurzen und schnellen Anhäufung von Gewinnen mit List und Skrupellosigkeit, dafür aber wenig Arbeit. Wenn Metaphern aus dem Bereich Kul- 
NATUR UND KULTUR ALS BILDSPENDER FÜR DIE KONZEPTUALISIERUNG...

tur zum Einsatz kommen, dann beziehen sie sich auf die Vertreter der Politik. Diese Metaphern sind zukunftsbezogen und bezeichnen Maßnahmen aus den Bereichen Landwirtschaft und Forstwirtschaft, mit denen der behauptete atavistische Zustand in der Wirtschaft kultiviert werden kann. Das erlaubt die Einsicht, dass Natur und Kultur nicht als Gegensätze, sondern vielmehr als miteinander komplementäre Begriffe zu verstehen sind. Die Natur liefert das Material. Was der Mensch daraus macht, ist Kultur (Hansen 2003: 29). Die Kultur-Metapher signalisiert dringenden Handlungsbedarf und eine Rückbesinnung auf die traditionellen Werte und Normen der Deutschen.

\section{LITERATURVERZEICHNIS}

Baldauf, C. (1997). Metapher und Kognition. Grundlagen einer neuen Theorie der Alltagsmetapher. Frakfurt am Main, Berlin, Bern, New York, Paris, Wien: Peter Lang.

Böke, K. (1996). Überlegungen zu einer Metaphernanalyse im Dienste einer "parzellierten" Sprachgeschichtsschreibung. In K. Böke, M. Jung, \& M. Wengeler, Öffentlicher Sprachgebrauch. Praktische, theoretische und historische Perspektiven. Wiesbaden: Westdeutscher Verlag, 431-452.

Busse, D. (2013). Linguistische Diskursanalyse. Die Macht der Sprache und die gesellschaftliche Konstruktion von Wirklichkeit aus der Sicht der linguistischen Epistemologie. In W. Viehöfer, R. Keller, \& W. Schneider, Diskurs-Sprache-Wissen. Interdisziplinäre Beiträge zum Verständnis von Sprache und Wissen in der Diskursforschung. Wiesbaden: Springer, 51-78.

Coulmas, F. (1992). Die Wirtschaft mit der Sprache. Eine sprachsoziologische Studie. Frankfurt am Main: Suhrkamp Verlag.

Foucault, M. (1981). Archäologie des Wissens. Frankfurt a. M.: Suhrkamp.

Hansen, K. P. (2003). Kultur und Kulturwissenschaft. Eine Einführung. Tübingen und Basel: A. Franke Verlag .

Hebel, F. (1987). Sprache der Wirtschaft. Eine kritische Leseübung in Klasse 10. Der Deutschunterricht 21 . 4, 58-72.

Heires, M., \& Nölke, A. (2011). Finanzkrise und Finanzialisierung. In O. Kessler, Die Politische Ökonomie der Weltwirtschaftskrise. VS-Verlag, 37-52.

Hermanns, F. (2012b). Linguistische Anthropologie. Skizze eines Gegenstandsbereichs linguistischer Mentalitätsgeschichte. In H. Kämper, A. Linke, \& M. Wengeler, Der Sitz der Sprache im Leben. Beiträge zu einer kulturanalytischen Linguistik. Berlin, Boston: De Gruyter, 37-67. 
Jasmina Mahmutović

Jäkel, O. (2003). Wie Metaphern Wissen schaffen: die kognitive Metapherntheorie und ihre Anwendung in Modell-Analysen der Diskursbereiche Geistestätigkeit, Wirtschaft, Wissenschaft und Religion. Hamburg: Verlag Dr. Kovač .

Keller, R. (2003). Distanziertes Mitleid: Katastrophische Ereignisse, Massenmedien und kulturelle Transformation. Berliner Journal für Soziologie (13) 3, 395 - 414.

Kosellek, R. (1982). Krise. In O. Brunner, R. Kossellek, \& W. Conze, Geschichtliche Grundbegriffe, Bd. 3. Stuttgart: Klett - Cotta, 617-650.

Kruse, V., \& Barrelmeyer, U. (2012). Max Weber. Eine Einführung. Konstanz und München: UKV Verlagsgesellschaft mbH.

Lakoff, G. (1993). The Contemporary Theory of Metaphor. In A. Ortony, Metaphor and Thought. Cambridge: Cambridge University Press, 202-251

Lakoff, G., \& Johnson, M. (2011). Leben in Metaphern. Konstruktion und Gebrauch von Sprachbildern. Heidelberg: Carl Auer Systeme Verlag.

Lüger, H.-H. (1995). Presseanalysen. Meinungsbetonte Texte (Teil IV). Beiträge zur Fremdsprachenvermittlung. Heft 29/155, 111 - 137.

Luhmann, N. (1999). Die Realität der Massenmedien.

Musolff, A. (1991). Krieg oder Hochzeit? Metapherngebrauch in der Wirtschaftsberichtserstattung 2/91. Institut für deutsche Sprache .

Ortony, A. (1975). Why Metaphors are Necessary and not Just Nice. Educational Theory 25(1), 45-53.

Paul, J. (2004). Das (Tier)-Konstrukt und die Geburt des Rassismus. Münster: Unrast.

Schwarz, M., \& Chur, J. (2007). Semantik. Ein Arbeitsbuch.

Schwarz-Friesel, M. (2014). „Hydra, Krake, Krebsgeschwür, Sumpf, Killer-GmbH, Franchise-Unternehmen und Nebelwolke" - Perspektivierung und Evaluierungvon islamistischem Terrorismus durch Metaphern im deutschen Pressediskurs nach 9/11. In M. Schwarz-Friesel, \& J. Kromminga, Metaphern der Gewalt. Konzeptualisierungen von Terrorismus in den Medien vor und nach 9/11. Tübingen: Narr Francke Attempto Verlag GmbH + Co., 51 -74.

Schwarz-Friesel, M. (2015). Metaphern und ihr persuasives Inferenzpotenzial. Konzeptualisierungen des islamistischen Terrorismus nach 9/11 im massenmedialen Diskurs. In C. Spieß, Metapher und Metonymie. Berlin: De Gruyter. 144 - 160.

Stötzl, H., \& Wengeler, M. (1995). Kontroverse Begriffe: Geschichte des öffentlichen Sprachgebrauchs in der Bundesrepublik Deutschland . Berlin, New York: Walter de Gruyter.

Verdicchio, D. (2006). Finanzökonomie im Film. Monstrosität als Inklus- 
NATUR UND KULTUR ALS BILDSPENDER FÜR DIE KONZEPTUALISIERUNG...

ionsmodus. kulturRevolution: Zeitschrift für angewandte Diskurstheorie $50,58-63$.

Ziem, A. (2008b). "Heuschrecken" in Wort und Bild. Zur Karriere einer Metapher. Muttersprache 118, 108-120.

Ziem, A. (2013a). Wozu Kognitive Semantik. In B. Teubert, Linguistische Diskursanalyse: neue Perspektiven. Wiesbaden: Springer, 217-242.

\title{
Jasmina Mahmutović \\ PRIRODA I KULTURA KAO METAFORE ZA KONCEPTUALIZACIJU POJMA „EKONOMIJA“
}

\author{
Rezime
}

Autor u ovom članku analizira metafore iz oblasti prirode i kulture korištene u njemačkim medijima za konceptualizaciju apstraktnog pojma „ekonomija“. Cilj autora je da sazna da li se ekonomija doživljava kao prirodni proces koji je van domašaja čovjeka, ili pak kao nešto što je čovjek stvorio svojim radom. Analiza je rađena metodama kognitivne semantike na korpusu od 156 članaka objavljena u tri vodeća njemačka medija u jeku ekonomske krize 2008/2009. Cilj istraživanja je da se odgovori na sljedeća pitanja: Da li u tekstovima prevladavaju metafore iz oblasti prirode ili kulture? Šta nam te metafore otkrivaju o poimanju ekonomije i učesnika u ekonomskom procesu? Na osnovu empirijske analize autor dolazi do zaključka da se ekonomija u njemačkoj javnost uglavnom doživljava kao prirodni proces, a ne kao djelo čovjeka. Ekonomija i njeni učesnici opisuju se vokabularom iz oblasti divljine, flore i faune, lova i darvinističke borbe za opstanak. Atavističko stanje u njemačkoj ekonomiji pripisuje se uticaju anglosasksonskog neoliberalizma koji se u Njemačkoj počeo širiti 1990tih godina. Sasvim drugačija slika se stvara kada su u pitanju politika i njeni predstavnici. Političku vlast karakteriše njena odlučnost da stane u kraj neoliberalističkim strujama. U ovoj oblasti dominiraju metafore iz oblasti šumarstva i poljoprivrede, čime se daje do znanja da se atavističko stanje u privredi može prevazići i vratiti na nivo kulture.

Ključne riječi: priroda, kultura, ekonomija, metafore. 